\title{
Investigations into the route of uptake and pharmacokinetics of intraperitoneally-administered monoclonal antibodies: I. Transdiaphragmatic blockade of the terminal lymphatics in the rat
}

\author{
Jeffrey S. Barrett1, 2*, Richard L. Wahl1, John G. Wagner², Raya Brown'1, and Susan J. Fisher1 \\ University of Michigan Medical Center, ${ }^{1}$ Division of Nuclear Medicine, ${ }^{2}$ Department of Internal Medicine and UpJohn Center \\ for Clinical Pharmacology, Ann Arbor, Michigan
}

Received 18 October 1989/Accepted 15 March 1990

\begin{abstract}
Summary. Recent studies on the intraperitoneal administration of radiolabeled monoclonal antibodies indicate that the diaphragm and, in particular, the lymphatics associated with the diaphragm are more involved in the transport of such high-molecular-mass moieties than was earlier suspected. The current study examines the role of the diaphragm in the i.p. transport of an IgG2a murine monoclonal antibody, 5G6.4, by observing the effect on the absorption of the antibody produced when the diaphragm has been scarred. Normal, sham-operated, and diaphragmatically scarred (abrasions made with 600-grade sandpaper) female Sprague Dawley rats $(150-250 \mathrm{~g}$ ) were administered intraperitoneal injections of ${ }^{125}$ labeled 5G6.4 in a volume of $2.0 \mathrm{~cm}^{3}$. Approximately $5 \mu \mathrm{g}$ antibody protein was administered in the individual $19-\mu \mathrm{Ci}$ injections per rat. Scarring was effective in partially blocking the amount of labeled antibody that crossed the diaphragm. Mean diaphragm levels (\% injected dose/g) of 125I-labeled 5G6.4 from the scarred group were $16.8 \%$ lower than values from the sham-operated rats and $37.2 \%$ lower than those from the control rats. The blockade was effective in slowing the appearance of the labeled antibody in the systemic circulation. The half-time to absorption was significantly prolonged in the scarred group; mean $t^{1 / 2}$ absorption values of $2.5 \mathrm{~h}$ for the control group, $5.3 \mathrm{~h}$ for the shamoperated group, and $9.6 \mathrm{~h}$ for the diaphragmatically blocked group were recorded. Scarring the diaphragm reduced the mean maximum blood concentration by $27.6 \%$ over the control group and $23.9 \%$ over the sham-operated group. The mean time to maximum blood concentration was lengthened by $93.0 \%$ over the control group and $35.3 \%$ over the sham-operated group due as a result of scarification. Presumably this impedence to absorption would increase the time that the radiolabeled antibody bathed the peritoneal space. The scarred group also had the
\end{abstract}

\footnotetext{
* H. Helfman Pharmacy Student Aid Fellow

Offprint requests to: Richard L. Wahl, University of Michigan Medical Center, Division of Nuclear Medicine, 1500 East Medical Center Drive, Ann Arbor, MI 48 109-0028, USA
}

largest "system mean residence time" $(162.5 \mathrm{~h})$ compared to the sham-operated $(147.9 \mathrm{~h})$ and control $(118.7 \mathrm{~h})$ groups. These values further verify the effect of surgery on the kinetics of the i.p. administered radiolabeled monoclonals. This work demonstrates that scarifying the diaphragm does alter the kinetics of the i.p. administered monoclonal antibodies and supports the concept that transdiaphragmatic lymphatic absorption is an important route of antibody clearance from the peritoneal cavity.

\section{Introduction}

It is known that the intraperitoneal monoclonal antibody delivery is a pharmacokinetically rational approach to the delivery of radioantibody to malignancies localized to the peritoneal cavity [20]. While rational, the mechanism of antibody absorption from this cavity has not been extensively studied. Absorption of fluid from the peritoneal cavity is carried out, at least in part, by the terminal lymphatics, called lacunae, located in the diaphragm [14]. Lacunae communicate with the peritoneal cavity via stomatal openings between mesothelial cells that lead into channels formed by the combined processes of the mesothelial and lacunar endothelial cells $[14,15]$. Dedrick et al. [3], in justifying a pharmacokinetic rationale for peritoneal drug administration in the treatment of ovarian cancer, reported that most drugs absorbed from the peritoneum enter the portal system with the transdiaphragmatic lymphatics contributing only modestly to absorption. In support of this statement the authors cite data from Popovich et al. [12] showing that the peritoneal permeability is roughly inversely proportional to the square root of molecular mass. The published range of those experiments, however, is $<10 \mathrm{kDa}$, far less than the $150 \mathrm{kDa}$ of an intact IgG. Wahl et al. [20] have shown that the rates of uptake of several i.p. administered IgG's, IgM's, and their fragments are statistically equivalent. This study covered a range of 


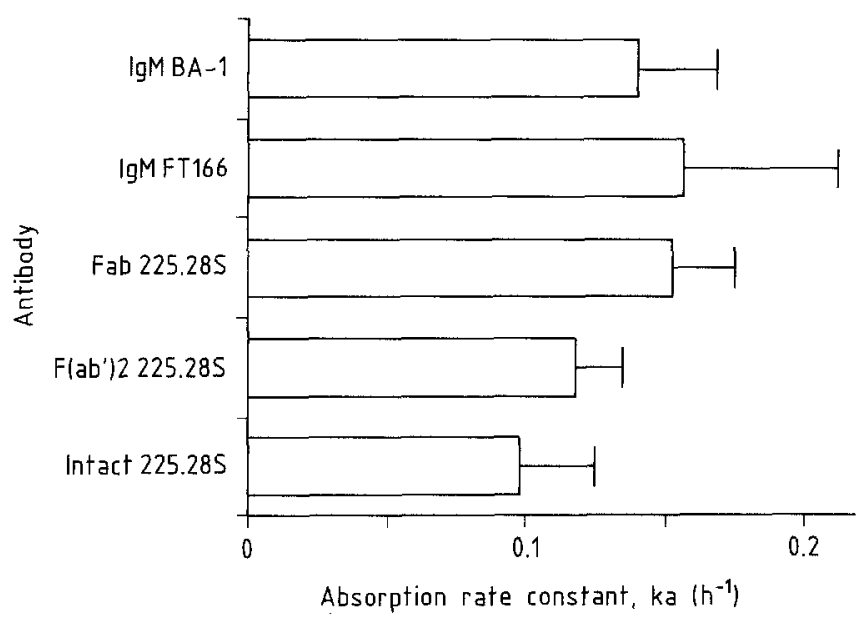

Fig. 1. Histogram from Wahl et al. [20] showing the equivalence of the absorption rate constants (mean \pm SD) of several i.p. administered radiolabeled monoclonal antibodies

molecular masses from $50 \mathrm{kDa}$ (Fab 225.28S) to $900 \mathrm{kDa}$ (IgM BA-1 and IgM FT166). Figure 1 is a histogram from those data showing the equivalence of the absorption rate constants of the antibodies studied [20]. Multiple comparison tests (Scheffe $F$-test and Dunnet $t$-test) found no difference between any of the groups [20]. Current unpublished data indicate further that the diaphragm and indeed the lymphatic system associated with this tissue are more involved in the egress of these antibodies from the peritoneal cavity than was earlier thought $[1,5,8]$ (and Barrett, Wahl and Wagner, unpublished data). Our ongoing studies to model the kinetics of intraperitoneal antibody delivery have shown that levels of radiolabeled antibody activity peak in the diaphragm and its draining lymphatics several hours before maximal blood levels are reached (Barrett et al. unpublished data). The early high levels in these tissues seem to indicate that the i. p. delivered radiolabeled monoclonal crosses the diaphragm to an appreciable level consistent with the diaphragmatic lymphatics contributing to a major extent to the egress of the antibody.

Tsilibary and Wissig [14] have shown that the patency of the diaphragm stomata can vary in response to changing

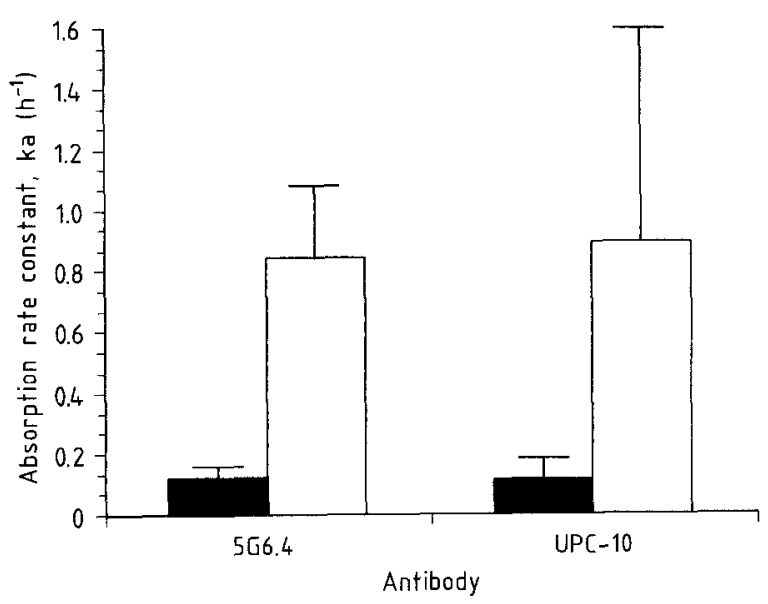

Fig. 2. Histogram from Wahl et al. [20] showing the effect of tumor burden on the absorption rate constants (mean $\pm S D$ ) of i.p. administered 5G6.4 and UPC-10 ( with tumor; $\square$ normal) conditions in the peritoneal cavity. Contractile components of the mesothelial and endothelial cells seem to be responsible for maintaining the conformation of both stomata and underlying channels and presumably also for effecting changes in the patency of stomata. They observed numerous patent stomata when the diaphragm is relaxed and when intraabdominal fluid pressure is raised (as with the addition of fluid from an i.p. injection) [14]. This appears to be an adaptive response to expedite removal of fluid from the peritoneal cavity. Also, ascites accompanying peritoneal parasitic infection resulted in hypertrophy of mesothelial cells and fewer patent stomata even though the peritoneal cavity was filled with abundant ascitic fluid and intraabdominal pressure had presumably increased [14]. This could explain, at least in part, the local effect of an i.p. "tumor burden" and why the absorption rate constant from the peritoneal cavity of tumor-laden mice was significantly $(P<0.005)$ different from that of normal mice (eight times smaller) in the findings of Wahl et al. [20]. Figure 2 (adapted from [20]) shows the effect of tumor burden on the absorption of two $\operatorname{IgG} 2$ a murine monoclonal antibodies, the ovarian cancer-"specific" IgG2ak 5G6.4 and the myeloma IgG2ak UPC-10, administered i.p.

The principal aim of this study is to determine whether the absorption of an antiovarian IgG2a (5G6.4) antibody is altered by scarification of the peritoneal surface of the diaphragm. The resulting fibrosis and scarring may induce a blockade of diaphragmatic absorption [4]. Therefore, if the terminal lymphatics are in fact responsible for a significant component of the absorption of antibodies given i.p., a significant difference in the absorption of the labeled antibody between the experimental and control groups should be detected. A third group (sham-operated) was added to determine whether there is a significant effect due to surgery alone, without diaphragmatic blockade, on any measurable effects associated with absorption into the systemic circulation. The main objectives of this study are to measure the extent to which the diaphragm is blocked by scarification, to demonstrate an improved i.p. delivery advantage in scarred animals over administration in nontreated animals, and to evaluate the effect of diaphragmatic blockade on tissue distribution. This could have therapeutic significance in that the regional delivery advantage of i.p. administration could potentially be further exploited by limiting the egress of antibody from the peritoneal cavity.

\section{Materials and methods}

Monoclonal antibodies. The mouse IgG2a monoclonal antibody, 5G6.4 was studied. $5 \mathrm{G} 6.4$ is known to react preferentially with ovarian and other epithelial cancers [19]. The antibody was purified from ascites using staphylococcal protein A chromatography [19]. The molecular mass and purity of the purified antibody were verified on $7.5 \%$ sodium dodecyl sulfate/polyacrylamide gels [7].

Radiolabeling. All labelings were conducted using ${ }^{125} \mathrm{I}$ (New England Nuclear, Boston, Mass), by the iodogen method (Pierce Chemical, Rockford, Ill) [10], $1 \mathrm{mCi}$ being used to label $100 \mu \mathrm{g}$ antibody protein. Free lodine was removed from the mixture after labeling by anion-exchange resin. lodine incorporation for this procedure is in the $50 \%-80 \%$ range. 
The specific activity of the administered injection mixture was approximately $4 \mu \mathrm{Ci} / \mu \mathrm{g}$. Freedom from iodine contamination was demonstrated by silica gel thin-layer chromatography with $50 \%$ ethyl acetate and $50 \%$ ethanol [18].

Administration. Two weeks prior to injection, 35 adult female SpragueDawley rats $(150-200 \mathrm{~g})$ were separated into three groups $(15$ experimental, 10 sham-operated, and 10 control rats). The 15 experimental and the 10 sham-operated rats had a small midline incision cut into the abdomen. The peritoneal surface of the diaphragm of the experimental group rats was gently rubbed with a moistened piece of 600-grade sandpaper [4]. Talc (U.S.P. 8476, Mallinckrodt Inc., St. Louis, MO) was then dusted onto the abraded surface as a further irritant to induce fibrosis. The sham-operated group was merely probed under the diaphragm with the forefinger. The peritoneal cavity of the treated animals was closed with 4-0 chromic suture. Closure of the outer wound was completed by stapling with automatic wound clips. Injection mixtures were prepared using a Capintec radioisotope calibrator (CRC-4R). Rats were given single $19-\mu \mathrm{Ci}$ injections of ${ }^{125} \mathrm{I}$-labeled $5 \mathrm{G} 6.4$. The individual doses were administered in a $2.0-\mathrm{cm}^{3}$ volume of saline at $37^{\circ} \mathrm{C}$. Rats were anesthetized with ether, weighed, and numbered prior to injection. The i.p. injections were administered with a 27 -gauge needle attached to a $3-\mathrm{ml}$ syringe. Rats that died during the study or in which the attempted i.p. injection was not successful were subsequently dropped from the study. Blood samples ( $\approx 13$ over time) were drawn from the tail vein through pasteur pipettes and weighed on glassine paper using a Mettler balance. Samples were then placed in $12 \times 75$ - $\mathrm{mm}$ test-tubes and capped. Rats were returned to the group cage overnight. At the end of the 7-day experiment, blood samples and the excised thyroid, anterior mediastinal lymph nodes, kidneys, and diaphragms were weighed and counted in a Packard 5780 gamma counter with the appropriate corrections for count decay. Several diaphragms from each group were removed for examination at the conclusion of the study. Perpendicular histological sections to the ventral plane of the diaphragm approximately $5 \mu \mathrm{m}$ thick were cut, stained with $\mathrm{H} \& \mathrm{E}$ and photographed. Examination of the radiolabeled components in the blood serum and i.p. fluid was carried out on three rats at $45 \mathrm{~min}, 2 \mathrm{~h}$, and $4 \mathrm{~h}$. Following centrifugation, sizing analysis of the supernatant was carried out on a Pharmacia FPLC system with a Superose 6 gel filtration column. Fractions were collected and counted on a Packard 5780 gamma counter.

Pharmacokinetics. Blood samples were collected over a 7-day period. The data were corrected for decay and plotted as time/activity curves for whole blood. Pharmacokinetic analysis was completed using RSTRIP [11]. The program employs a nonlinear least-squares subroutine in its fitting module. A weighting factor of $1 / y$ was used for each fit. For each time course exhaustive curve strippings up to four exponentials were generated. Those which gave physiologically impossible parameters (incorrect sign in front of coefficient or exponent) were subsequently dropped from the model comparison. The remainder were used as initial

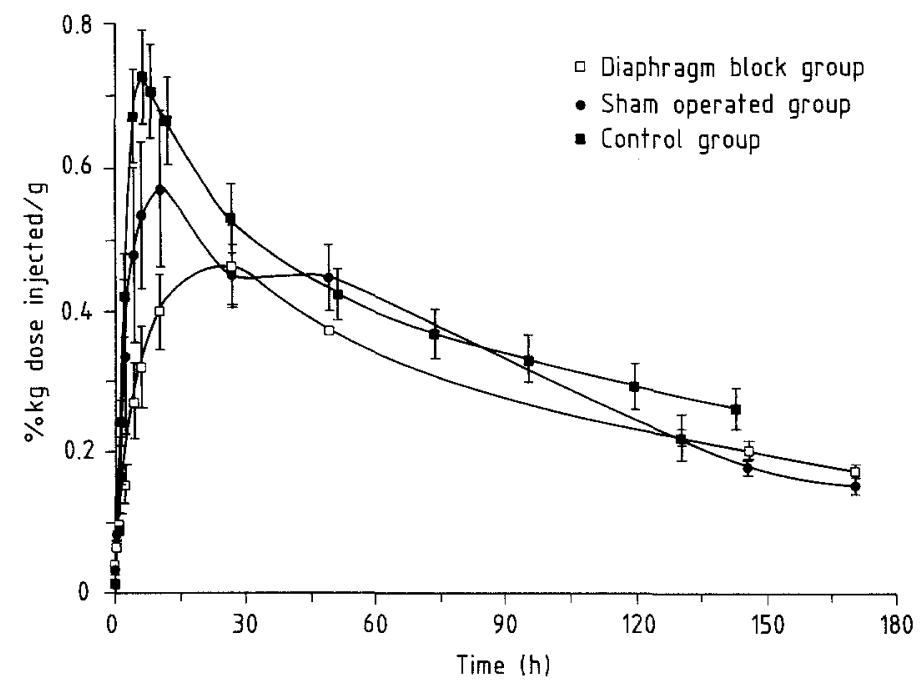

Fig. 3. Mean blood levels (mean \pm SEM) from i.p. administered ${ }^{125}$ I-labeled $5 \mathrm{G} 6.4$ in three treatment groups of female Sprague Dawley rats $(150-250 \mathrm{~g})$

estimates for fitting using nonlinear least-squares and the associated $F$-tests (MSC, model selection criteria in RSTRIP), $r^{2}$, and parameter standard errors were evaluated for the best fits. Individual rat blood level curves were fitted and modeled from the $\% \mathrm{~kg}$ dose $/ \mathrm{g}$ units (Units of concentration based on the $\%$ of the original injected counts available in the blood at time t, normalized to the weight of the animal). The parameterized values were then converted into standard pharmacokinetic units. Mean data were used to generate the histograms in Results.

Statistical analysis. SYSTAT [13] was used to complete the individual analyses of variance and tests for homogeneity of variance. Linear contrasts from the original one-way analyses of variance were made for partial comparisons across pairs of treatment groups. Box and whisker plots were made for the volume of distribution, blood clearance, and absorption phase half-life.

\section{Results}

\section{Experimental}

Mean blood level curves of 125I-labeled 5G6.4 for the three treatment groups are shown in Fig. 3. The integrity of the $125 \mathrm{I}$-labeled $5 \mathrm{G} 6.4$ species in vivo is demonstrated by

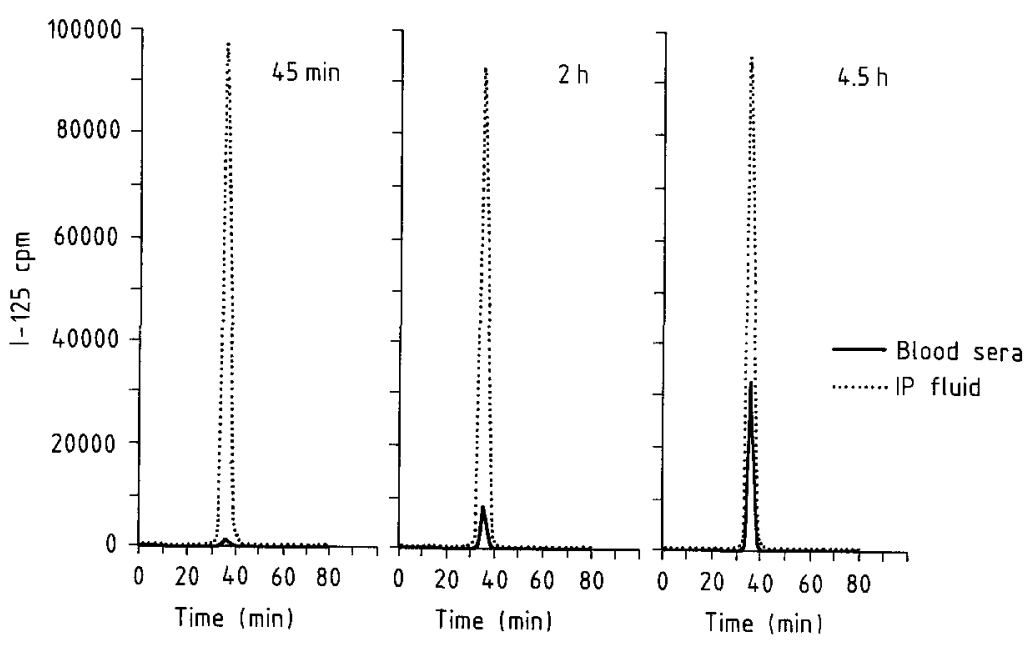

Fig. 4. Activity profiles of the ${ }^{125}$ I-labeled $5 \mathrm{G} 6.4$ species at $45 \mathrm{~min}, 2 \mathrm{~h}$, and $4 \mathrm{~h}$ after i.p. injection observed in the serum and intraperitoneal fluid by FPLC radiochromatog raphy 

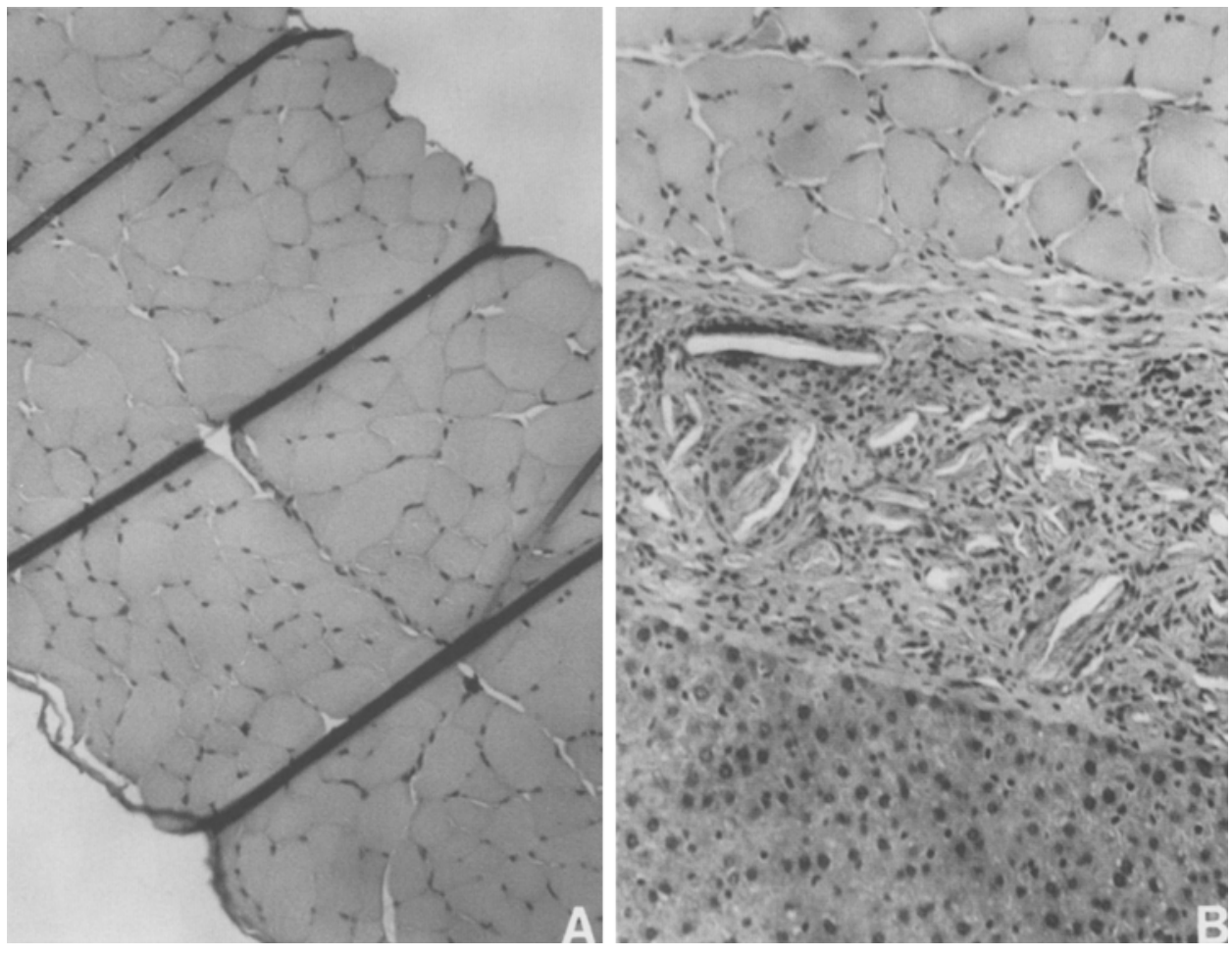

Fig. 5 A, B. Perpendicular histological slices of rat diaphragm $(10 \times)$ excised at sacrifice (7 days) after i.p. administration of ${ }^{125}$ I-labeled 5G6.4. A Normal rat diaphragm. B Diaphragm (top) from scarified rat showing granulation layer formed due to scarring (middle) and attached normal liver (bottom)

Fig. 4. Early serum samples examined by FPLC radiochromatography show no indication of any secondary 125I-labeled metabolic products. This verifies the work of Himmelsbach et al. [6] with 125I-labeled 5G6.4. Later assays in the serum, kidneys, and liver also show no evidence of formation of species of either higher or lower molecular mass after i.p. administration of 125I-labeled 5G6.4 though the urine contains free iodine [6]. The blood levels (see Fig. 3) indicate that there is a delayed appearance of the labeled antibody in the scarified group. Moreover, on the basis of the mean blood levels, the maximum concentrations of the diaphragmatically blocked and the shamoperated group were lower than those of the control group. Finally, the ascent of the absorption phase is much steeper in the control and sham groups than in the diaphragmatically blocked group. The pharmacokinetics section, which follows, gives a more detailed analysis of the parameterized blood levels.

Figure 5 shows a photomicrograph of a histological section (10 $\times$ magnification) of a diaphragm from one of the control group rats (A) and a scarified diaphragm (B), showing the granulation layer formed from the fibrosis due to scarring and the adhesion of this layer to the liver. The tissue was cut perpendicular to the ventral plane. The adhesion appears to be caused by the thick granulation tissue containing particles of talc and sand that were used for scarification. Notice that the diaphragm itself appears normal.

Figure 6 shows a histogram of mean 125I-labeled 5G6.4 levels in the kidney, anterior mediastinal lymph nodes, and diaphragm with their respective standard error bars. The scarified group had significantly lower levels in the kidney $(P=0.027)$ and diaphragm $(P=0.048)$ than the other two groups (control and sham). While the measured levels in the lymph nodes were lower in the scarified and shamoperated groups, they were statistically equivalent to those of the control group. Mean radioantibody levels in the thyroid are shown in Fig. 7. These levels represent mostly free iodine. There exists a statistically significant difference $(P<0.001)$ between the diaphragmatically-blocked and the other two groups. This may be due to slower antibody egress from the peritoneal cavity. Longer exposure of the labeled compound may increase the total amount of deiodination in the liver or peritoneal cavity.

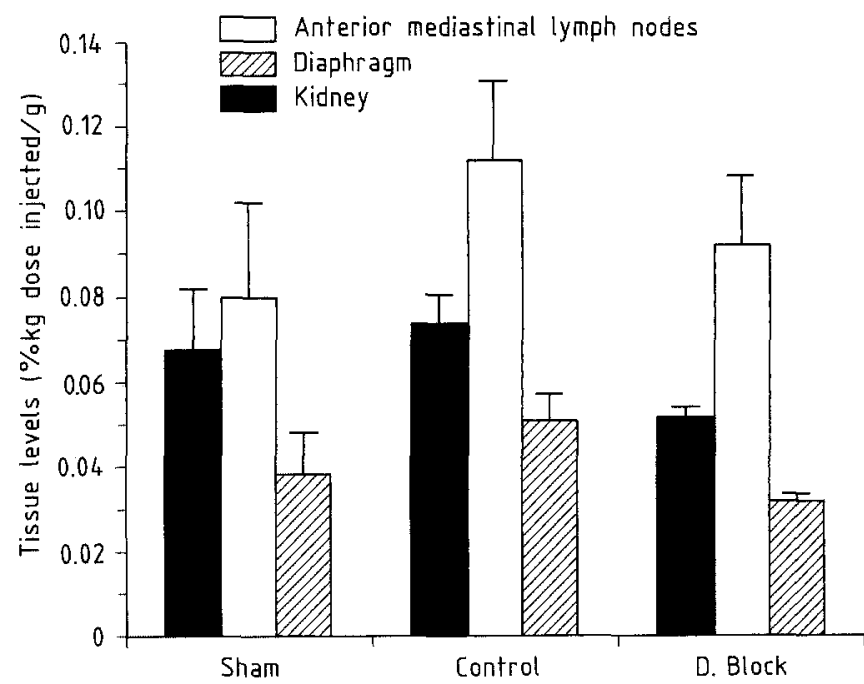

Fig. 6. Mean tissue levels (mean \pm SEM) from i. p. administered ${ }^{125} \mathrm{I}-\mathrm{la}$ beled $5 \mathrm{G} 6.4$ in three treatment groups of female Sprague Dawley rats $(150-250 \mathrm{~g}) 7$ days post injection 


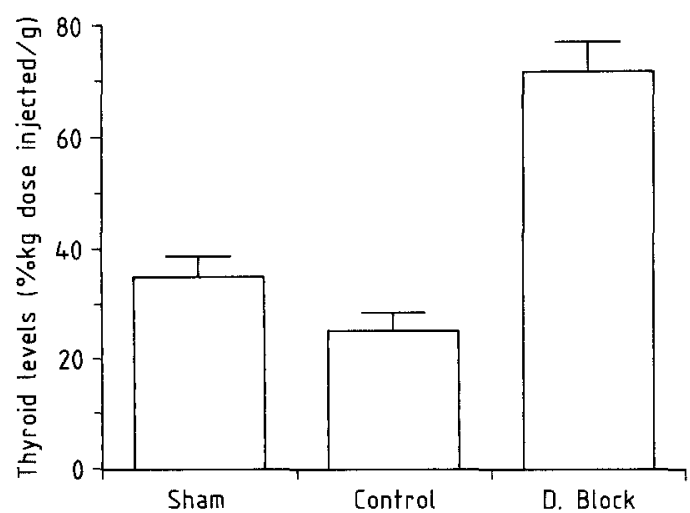

Fig. 7. Mean thyroid levels (mean \pm SEM) from i.p. administered ${ }^{125} \mathrm{I}-$ labeled 5G6.4 in three treatment groups of female Sprague Dawley rats $(150-250 \mathrm{~g}) 7$ days post injection

\section{Pharmacokinetic modeling}

Individual subject (rats) sets of concentration ( $\mathrm{kg} \%$ dose injected /g)/time data were fitted for each antibody in every rat to a bi-exponential curve using the method of leastsquares in the program RSTRIP [11]. Initial estimates were obtained by the curve-stripping routine of the same program. Below is the schematic diagram of the standard one-compartment open model used to fit each blood/time curve. The equations are straightforward as follows.

$C=\left(\frac{\mathrm{FD}}{V_{\mathrm{d}}}\right)\left(\frac{k_{\mathrm{a}}}{k_{\mathrm{a}}-K}\right)\left(\mathrm{e}^{-K t}-\mathrm{e}^{-k \mathrm{a} t}\right)$

where $\mathrm{FD} / V$ is analogous to $C_{0}$ (blood concentration at time $=0$ ) after intravenous administration. $K$ is the overall elimination rate constant for the labeled antibody or the fractional rate of labeled antibody elimination.

$K=\frac{\text { Rate of elimination }}{[\mathrm{Ab}]}=\frac{C L}{V_{\mathrm{d}}}$

The rate of elimination can be written as follows:

$-\frac{\mathrm{d}[\mathrm{Ab}]}{\mathrm{d} t}=C L C$

where $[\mathrm{Ab}]$ is the amount in the body, $V_{\mathrm{d}}$ is the volume of distribution in the plasma compartment, $C L$ is the blood clearance (volume/time), and $C$ is the blood concentration. Integrating from 0 to infinity yields.

$-\int_{0}^{\infty} \mathrm{d}[\mathrm{Ab}]=C L \int_{0}^{\infty} C \mathrm{~d} t$

From here an expression for blood clearance can be derived.

$$
\frac{C L}{\mathrm{~F}}=\frac{\text { i.p. dose }}{(\mathrm{AUC})_{0 \rightarrow \infty}}
$$

where $\mathrm{F}$ is the bioavailability or the fraction of the dose that is actually absorbed, and AUC is the area under the curve. Hence, the blood concentration of these two antibodies can be explained by the classical one-compartment open model with first-order input (see Scheme):

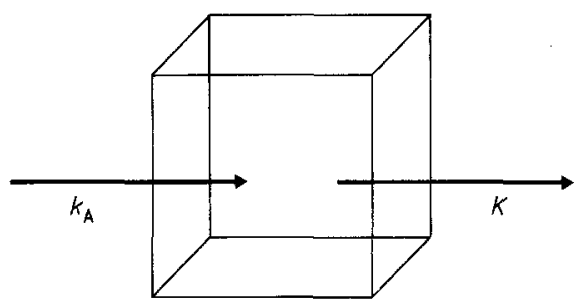

Mean residence time (MRT) parameters are often used to measure the variation in steady-state blood concentrations [16] and to approximate the amount of systemic exposure of the drug and/or antibody in question $[1,16]$. It is the latter that we are concerned with here. The system MRT is the mean residence time during which the compound remains in the entire model - the body.

System MRT $=\frac{\text { AUMC }_{0 \rightarrow \infty}}{\mathrm{AUC}_{0 \rightarrow \infty}}$

AUMc is the area under the first-moment curve. The plasma MRT is the mean residence time in which the compound remains in the central or plasma compartment.

Plasma MRT $=\frac{\mathrm{AUC}_{0 \rightarrow \infty}}{C_{0}}=\frac{1}{k_{\mathrm{el}}}$

where $k_{\mathrm{el}}$ is the elimination rate constant from the plasma compartment. For the one-compartment model, kel equals the overall first-order elimination rate constant, $K$, since elimination is assumed to be from the plasma compartment only.

Table 1 contains the mean pharmacokinetic parameters derived from the blood levels of rats of each group. The sample size of each group is included with the results of a one-way analysis of variance for two levels of confidence (90\% and 95\%). In addition, partial comparison tests (linear contrasts made on the $C$-matrix from the original analyses of variance) are included for each parameter. The symbols $=$ and $\neq$ under the partial comparison heading refer to statistical equivalence and significant difference respectively. The $P$-values associated with these linear contrasts refer to the probability of the null hypothesis the probability that the two groups in the contrast are equivalent.

It is evident, as Table 1 indicates, that indeed there is an effect of surgery with respect to the kinetics of 125 I-labeled 5G6.4 administered intraperitoneally. Both the diaphragmatically blocked and sham-operated groups clear the blood more slowly $(0.123 \mathrm{ml} / \mathrm{h}$ and $0.138-0.185 \mathrm{ml} / \mathrm{h})$ and values are distributed to a lesser extent $\left(V_{\mathrm{d}}=14.8 \mathrm{ml}\right.$ and $14.74-20.71 \mathrm{ml}$ ) than those of the non-operated control group. However, the plasma MRT values of all three groups are statistically equivalent. This seems to indicate 
Table 1. Pharmacokinetic parameter summary and statistical inference across treatment groups

\begin{tabular}{|c|c|c|c|c|c|c|}
\hline \multirow[t]{2}{*}{ Mean parameter ${ }^{\mathrm{a}}$} & \multicolumn{3}{|c|}{ Treatment group } & \multicolumn{3}{|c|}{ Analysis of variance significance results } \\
\hline & $\begin{array}{l}\text { Control } \\
(n=8)\end{array}$ & $\begin{array}{l}\text { Diaphragmatic } \\
\text { block } \\
(n=11)\end{array}$ & $\begin{array}{l}\text { Sham } \\
(n=7)\end{array}$ & $\alpha=0.05$ & $\alpha=0.1$ & Partial comparison tests \\
\hline$t^{1 / 2}$ absorption (h) & $\begin{array}{c}2.49 \\
(0.27)\end{array}$ & $\begin{array}{c}9.59 \\
(1.83)\end{array}$ & $\begin{array}{c}5.34 \\
(0.88)\end{array}$ & $\begin{array}{l}\text { Yes } \\
(P=0.005)\end{array}$ & Yes & $\begin{array}{l}\text { Control }=\text { sham; }(P=0.203 \\
\text { Control } \neq \text { d.block; } \alpha=0.05 ; p=0.001 \\
\text { Sham } \neq \text { d.block; } \alpha=0.05 ; P=0.047\end{array}$ \\
\hline Time at $C_{\max }(\mathrm{h})$ & $\begin{array}{l}12.72 \\
(0.97)\end{array}$ & $\begin{array}{l}24.55 \\
(4.36)\end{array}$ & $\begin{array}{l}18.15 \\
(4.37)\end{array}$ & No & $\begin{array}{l}\text { Yes } \\
(P=0.070)\end{array}$ & $\begin{array}{l}\text { Control }=\text { sham } P=0.546 \\
\text { Control } \neq \text { d.block; } \alpha=0.05 ; P=0.028 \\
\text { Sham = d.block; } P=0.125\end{array}$ \\
\hline$C_{\max }(\% \mathrm{~kg}$ dose inj $/ \mathrm{g})$ & $\begin{array}{c}0.670 \\
(0.058)\end{array}$ & $\begin{array}{c}0.485 \\
(0.030)\end{array}$ & $\begin{array}{c}0.637 \\
(0.075)\end{array}$ & $\begin{array}{l}\text { Yes } \\
(P=0.029)\end{array}$ & Yes & $\begin{array}{l}\text { Control }=\text { sham; } P=0.669 \\
\text { Control } \neq \text { d.block; } \alpha=0.05 ; P=0.014 \\
\text { Sham } \neq \text { d.block; } \alpha=0.05 ; P=0.048\end{array}$ \\
\hline$V_{\mathrm{d}} / F(\mathrm{ml})$ & $\begin{array}{l}20.71 \\
(2.53)\end{array}$ & $\begin{array}{l}14.85 \\
(1.45)\end{array}$ & $\begin{array}{l}14.74 \\
(3.02)\end{array}$ & No & $\begin{array}{l}\text { Yes } \\
(P=0.125)\end{array}$ & $\begin{array}{l}\text { Control } \neq \text { sham; } \alpha=0.1 ; P=0.089 \\
\text { Control } \neq \text { d.block; } \alpha=0.1 ; P=0.065 \\
\text { Sham = d.block; } P=0.971\end{array}$ \\
\hline$C L / F(\mathrm{ml} / \mathrm{h})$ & $\begin{array}{c}0.185 \\
(0.025)\end{array}$ & $\begin{array}{c}0.123 \\
(0.007)\end{array}$ & $\begin{array}{c}0.138 \\
(0.012)\end{array}$ & $\begin{array}{l}\text { Yes } \\
(P=0.022)\end{array}$ & Yes & $\begin{array}{l}\text { Control } \neq \text { sham; } \alpha=0.1 ; P=0.057 \\
\text { Control } \neq \text { d.block; } \alpha=0.05 ; P=0.007 \\
\text { Sham }=\text { d.block; } P=0.496\end{array}$ \\
\hline System MRT (h) & $\begin{array}{r}118.72 \\
(8.90)\end{array}$ & $\begin{array}{l}162.51 \\
(16.00)\end{array}$ & $\begin{array}{l}147.88 \\
(13.63)\end{array}$ & No & No & $\begin{array}{l}\text { Control }=\operatorname{sham} ; P=0.192 \\
\text { Control } \neq \text { d.block; } \alpha=0.05 ; P=0.034 \\
\text { Sham = d.block; } P=0.478\end{array}$ \\
\hline Plasma MRT (h) & $\begin{array}{r}115.15 \\
(8.86)\end{array}$ & $\begin{array}{l}122.13 \\
(11.37)\end{array}$ & $\begin{array}{l}100.33 \\
(16.67)\end{array}$ & No & No & $\begin{array}{l}\text { Control = sham; } P=0.438 \\
\text { Control = D.block; } P=0.683 \\
\text { Sham = d.block; } P=0.227\end{array}$ \\
\hline
\end{tabular}

a $V_{\mathrm{d}}$, volume of distribution in plasma compartment; $C_{\max }$, maximum blood concentration; $C L$, blood clearance; F, bioavailability (fraction of dose absorbed); MRT, mean residence time

that there exists a difference in tissue and/or plasma protein binding between the operated and non-operated groups. The system MRT in the scarified group is greater than in the other two groups, yet not significantly different from that of the sham-operated group. It may be that adhesions due to surgery are an added site of binding or at least of transient retardation of entry to the systemic circulation of the labeled $\mathrm{mAb}$ and therefore the operated groups exhibited longer "whole model" residence times. As the

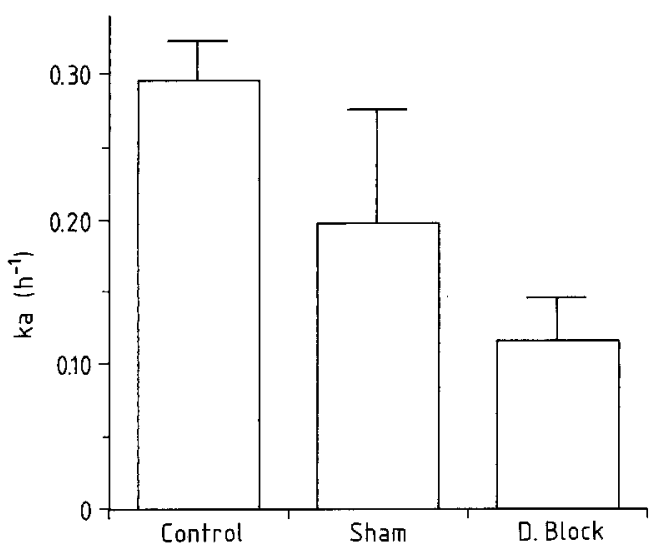

Fig. 8. Mean absorption tate constants (mean \pm SEM) across treatment groups from i.p. administered ${ }^{125}$ I-labeled 5 G6.4 female Sprague Dawley rats mean blood levels (see Fig. 3) indicate, the time to maximum blood concentration is longer and the maximum blood concentration is smaller in the diaphragmaticallyblocked group than in the other two groups. The actual values can be found in Table 1. Yet, for both parameters, the sham-operated group appears to be between the control and scarified groups. Statistically, the sham-operated group is equivalent to the control group with respect to time at $C_{\max }$ and equivalent to the scarified group with respect to $C_{\max }$. The most dramatic result of this procedure lies in the $t_{1 / 2}$ absorption values (see Fig. 8). The shamoperated and control groups are statistically equivalent (mean values of $2.49 \mathrm{~h}$ and $5.38 \mathrm{~h}$ ), but the group with the diaphragmatic block $(9.59 \mathrm{~h})$ is significantly different from both these groups.

With respect to the analysis of these data, the mean values, although the standard errors are included, are not always informative enough to explain the results from an analysis of variance. Figure 9 a, b, c shows box and whisker plots for blood clearance, $t_{1 / 2}$ absorption, and volume of distribution. The plots illustrate the effect of the spread of the data on the conclusions from the analysis of variance and subsequent pairwise comparisons. Figure $9 \mathrm{~b}$ shows the spread of the $t_{1 / 2}$ absorption values for each group relative to their means. Even with the larger variation in the scarified group, it was found to be significantly different from both of the other groups at a $95 \%$ confidence level. Asterisks, as in Fig. 9 a, denote outliers to the $\mathrm{H}$-spread of the plot. 

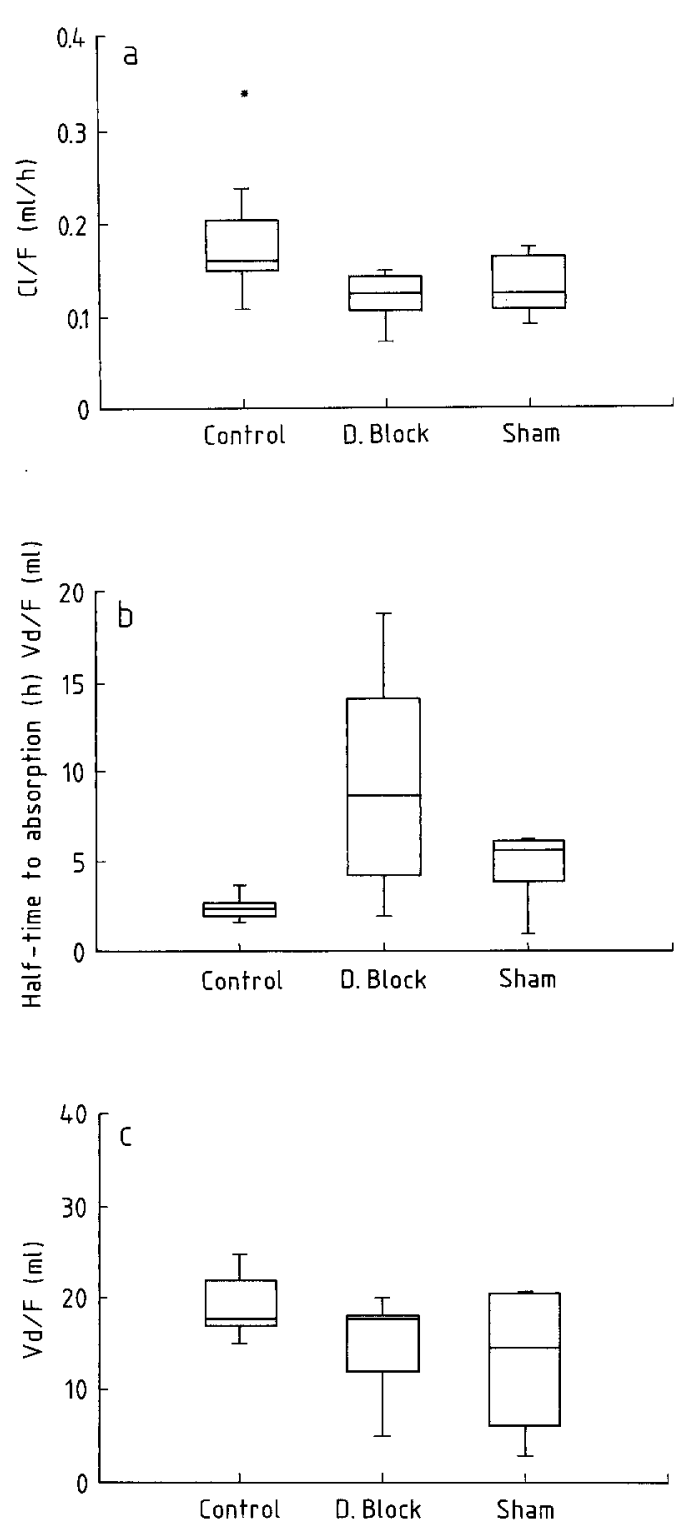

Fig. 9 a -c. Box and whisker plot of (A) blood clearance values, (B) $t_{1 / 2}$ absorption values, and $(\mathbf{C})$ volume distribution values across treatment groups

\section{Discussion}

The present study indicates that the kinetics of distribution of i.p. delivered radiolabeled murine $\mathrm{IgG} 2 \mathrm{a}$ monoclonal antibody $5 \mathrm{G} 6.4$ can be altered by scarification of the diaphragm. This approach significantly slows the rate of absorption of the labeled antibody, delays the time to its peak blood level, and reduces the magnitude of these peak levels. These effects are almost certainly due to a high level of obstruction of the entrance to the transdiaphragmatic lymphatics by scarring, as can be seen by light microscopy. While it is known that swelling of the mesothelial cells of the diaphragm due to infection can result in the closure of stomatal openings, infections in the operated groups were not observed. The less marked effects in the sham-operated group and the control group add further support to the concept that the diaphragm has a significant role in the mechanism of antibody uptake from the peritoneal cavity. Additional studies with more tissues at earlier time points and with other antibodies may increase our understanding.

These experimental observations indicate that the transdiaphragmatic absorption of antibodies is making a substantial contribution to the total radioantibody absorption from the peritoneal cavity. This is in contrast to prior studies suggesting that transperitoneal membrane absorption is the major route of antibody egress. The absorption rate constants we observe are significantly higher than those predicted by permeability area product/molecular mass functionalities $[3,9]$. While the design of our experiment does not specifically address the relative contribution to absorption from each route, it is also possible that this is not a constant ratio. Specifically, variations in i.p. fluid volume and pressure may result in varying stomatal opening, which may alter the proportion of antibody leaving through the diaphragmatic lymphatics. More studies are necessary and are in progress.

It is possible that the delayed i.p. fluid absorption caused by diaphragmatic blockade may offer a significant improvement in the regional delivery advantage of i.p. administration to intraperitoneal tumors by increasing the time these tumors are bathed with the radioantibody [20]. Further, if combined with peritoneal lavage for therapeutic use, additional improvements in the regional delivery advantage might be made [17]. Certainly, our invasive procedure is not an option for human therapy, though i.p. carcinomatosis by itself may block the lymphatics and the flow of fluid from the peritoneal cavity $[2,8,17]$. Possibly, an alternative method of blocking the diaphragm will increase the time that the labeled compound is exposed to the peritoneal cavity and thus increase the regional delivery advantage offered by i.p. administration. Even if not immediately applicable clinically, this study strongly supports our hypotheses regarding the importance of the diaphragmatic lymphatics in the physiology of peritoneal transport of high-molecular-mass protein species.

Acknowledgements: The technical assistance of Andrew Kucharski, Tom Gutowski, Chris Henry, and Gayle Jackson is much appreciated. Supported by CA 41531 and CA 33802 awarded by the P. H. S.

\section{References}

1. Albert A, Takamatsu H, Fonkalsrud EW (1984) Absorption of glucose solutions from the peritoneal cavity in rabbits. Arch Surg 119: $1247-1251$

2. Barrett JS, Fisher S, Wagner JG, Wahl RL (1989) Enhanced regional delivery of monoclonal antibodies to the peritoneal cavity by transdiaphragmatic blockade. V Nucl Med 30: 815 (abstr).

3. Dedrick RL, Myers CE, Bungay PM, DeVita Jr VT (1978) Pharmacokinetic rationale for peritoneal drug administration in the treatment of ovarian cancer. Cancer Treat Rep 62: 9

4. Dumont AE, Maas WK, Ilieuscu H, Shin RD (1986) Increased survival from peritonitis after blockade of transdiaphragmatic absorption of bacteria. Surg Gynecol Obstet 162: 248-251

5. Flessner MF, Fenstermacher JD, Blasberg RG, Dedrick RL (1984) Peritoneal absorption of macromolecules studied by quantitative autoradiography. Am J Physiol 248: H26-H32

6. Himmelsbach M, Wahl RL (1989) Studies on the metabolic fate of ${ }^{111}$ In-labeled antibodies. Int J Rad Appl Instrum [B] 16: 839-845 
7. Laemmli VK (1970) Cleavage of structural proteins during assembly of the head of bacteriophage T4. Nature 222: 680-685

8. Levine S (1985) Postinflammatory increase of absorption from peritoneal cavity into lymph nodes: particulate and oily inocula. Exp Mol Pathol 43: $124-134$

9. Leypoldt JK, Parker HR, Frigon RP, Henderson LW (1987) Molecular size dependence of peritoneal transport. J Lab Clin Med 110: 207-216

10. Markwell MAK (1982) A new solid-state reagent to iodinate proteins: I. Conditions for the efficient labeling of antiserum. Anal Biochem 125: $427-432$

11. MicroMath Scientific Software 2034 East Fort Union Blvd, Salt Lake City, UT 84121-3144

12. Popovich RP, Moncrief JW, Decherd JF, et al. (1977) Physiological transport parameters in patients in peritoneal and hemodialysis. In: Proceedings of the 10th Annual Contractor's Conference: Artificial Kidney-Chronic Uremia Program. National Institute of Arthritis, Metabolism and Digestive Diseases, pp 95-98

13. SYSTAT Inc. 1800 Sherman Ave. Evanston, Illinois

14. Tsilibary EC, Wissig SL (1983) Lymphatic absorption from the peritoneal cavity: regulation of patency of mesothelial stomata. Microvasc Res 25: 22-39
15. Verger C, Luger A, Moore HL, Nolph KD (1983) Acute changes in peritoneal morphology and transport properties with infectious peritonitis and mechanical injury 23: 823-831

16. Wagner JG (1988) Types of mean residence times. Biopharm Drug Dispos 9: $51-57$

17. Wahl RL, Liebert M (1989) Improved radiolabeled monoclonal antibody uptake by lavage of intraperitoneal carcinomatosis in mice. $J$ Nucl Med 30: 60-65

18. Wahl RL, Liebert M, Carey JE, Jackson G (1985) Quality control of radiolabeled monoclonal antibodies: immunologic and radiochemical. Cancer Drug Deliv 2: 236

19. Wahl RL, Liebert M, Biesman B, Roberts J, Jackson G, Kronberg S, Laino L (1986) Production and characterization of a murine monoclonal antibody reactive with ovarian and other epithelial carcinomas. Proc Am Assoc Cancer Res 27: 355

20. Wahl RL, Barrett JS, Geatti O, Licbert M, Wilson BS, Fisher S, Wagner JG (1988) The intraperitoneal delivery of radiolabeled monoclonal antibodies: studies on the regional delivery advantage. Cancer Immunol Immunother 26: 187-201 